\title{
Are the Boundary-Related Cells in the Subiculum Boundary-Vector Cells?
}

\author{
Dori Derdikman \\ Kavli Institute for Systems Neuroscience and Centre for the Biology of Memory, Medical-Technical Research Centre, Norwegian University of Science and \\ Technology, NO-7489 Trondheim, Norway \\ Review of Lever et al.
}

Lever et al. (2009) have made an endeavor to corroborate a prominent theory for the formation of place cells, the boundaryvector cell model (O'Keefe and Burgess, 1996; Hartley et al., 2000). They have recorded boundary-responsive cells in the subiculum, and claimed that these are boundary-vector cells. In this essay I discuss their claim. First, I suggest an analysis to highlight the boundary-related properties of these cells. Second, I argue with the authors' discussion about boundaryvector cells in the subiculum being inputs to place cells. As I will demonstrate, the anatomy of this system shows that this is most likely not the case.

More than 10 years ago, John O'Keefe and Neil Burgess showed how hippocampal place cells transform their firing fields when a small square box is turned into a larger rectangle, and then into a larger square box (O'Keefe and Burgess, 1996). They found that in some cases the place fields are anchored to one or two of the walls, such that when the expansion occurs, the cell's position continues to be determined by the distance from that wall. In other cases the cell's firing field split

Received Aug. 25, 2009; revised Sept. 9, 2009; accepted Sept. 9, 2009

This work was supported by the Kavli Foundation and a Centre of Excellence grant from the Norwegian Research Council. I would like to thank Torkel Hafting, Edvard I. Moser, Trygve Solstad, Jonathan R. Whitlock, and Menno P. Witter for reading and commenting on this manuscript.

Correspondence should be addressed to Dori Derdikman at the above address. E-mail: dori.derdikman@ntnu.no.

DOI:10.1523/JNEUROSCI.4176-09.2009

Copyright $\odot 2009$ Society for Neuroscience $\quad$ 0270-6474/09/2913429-03\$15.00/0 into two parts after the expansion of the box such that one of the split fields conserved the distance to one wall while the other field conserved the distance to the other parallel wall. Following these observations, they suggested that there are neurons which encode the perpendicular distance from walls. According to their idea, such hypothetical wall-distance cells act as inputs to place cells, which are suggested to be linear combinations of such cells. Later, Hartley and coworkers extended the description of these cells by suggesting that they are responsive to wall elements at a certain distance and direction from the rat (Hartley et al., 2000). These hypothetical cells were termed "boundary vector cells." For example, imagine such a hypothetical cell that fires when there is a wall $10 \mathrm{~cm}$ away from the rat, in the northwest direction from it. This same specific cell will not fire when there is a wall $10 \mathrm{~cm}$ away from the rat in the south direction. A schematic of such cells can be found in the study by Lever et al. (2009), their Figure 1. For many years it has not been known whether such cells indeed exist. Colin Lever and coworkers report such cells in the subiculum of the awake behaving rat (Lever et al., 2009).

They recorded extracellularly while rats were foraging in a square box, and noticed that a large proportion of the recorded cells (up to $24 \%$ ) demonstrated response characteristics reminiscent of boundary vector cells [Lever et al. (2009), their Fig. 2]. These cells expressed a graded firing rate as a function of the distance of the rat from one or two of the walls in the box. Usually the strongest response was near one of the walls, but some cells fired along a strip that was farther away from the wall [Lever et al. (2009), their Fig. 2E]. In all cases, Lever et al. (2009) tried to fit an optimal boundaryvector tuning curve to each of the cells (that is, to determine what is the optimal distance and direction of the wall for each cell), which demonstrated that the majority of boundary vectors were short, such that the cells fired only when the rat was very close to one of the walls [Lever et al. (2009), their Fig. $2 A-C$ ].

To test their idea of boundary vector cells, it is important to check what happens to these cells under various manipulations of the rat's environment. The prediction is that the cells should retain their boundary-vector properties when the environment is changed. Cells that fire at a certain distance from a wall that is east of the rat in a square environment should continue to fire when there is a wall east of the rat also in a round environment, in a tilted box, or in a box with an additional inserted wall [Lever et al. (2009), their Fig. $1 D]$. Most cells included in the paper behaved exactly like that. Consider for example cell $5 \mathrm{n}$ in Figure $3 \mathrm{C}$ of Lever et al. (2009). It is very similar to their illustration example, Figure $1 D$. Some other examples seem to behave in a similar way. Consider for example Lever et al.'s (2009) Figure $3 E$. This cell responded when there 
was a wall south of the rat, regardless of whether this was an outer wall of the box, or a wall that was inserted into the center of the box. Other examples, though, did not transform as well between environments. For example, cell $5 \mathrm{~m}$ in Lever et al.'s (2009) Figure 3C responded when there was a wall east of the rat in the square box. However, when the box was tilted on the side, or when it was transformed into a circular arena, the cell started to respond to a wall in the north-east direction instead. It would have been interesting to check also the response of more of these cells when the environment is double in size, similar to the experiment in O'Keefe and Burgess (1996).

To try to determine the shape of the boundary vector, I calculated "boundary rate plots" from the original rate maps in the study by Lever et al. (2009). Instead of plotting the firing rate as a function of the rat's position in the box, as in Lever et al.'s (2009) Figure 3, the firing rate of the cell as a function of the position of a wall element relative to the rat was plotted, in rat egocentric coordinates. Each $x-y$ point is an average of all points in the rate map which have a wall at a certain $x$-distance and $y$-distance from the rat (Fig. 1A). The four cells analyzed this way demonstrated somewhat similar responses to boundaries when moving from one environment to another (Fig. $1 B$ ). The differences between the plots can be explained by the effect of wall shape on pixel dependencies within each plot. That is, a straight wall tends to create straight responsive regions within the plot while a circular wall does not. Cell 1a fired maximally when there was an adjacent wall element southeast of the rat; cell 1c fired maximally when there was an adjacent wall element northwest of the rat; cell $5 \mathrm{n}$ fired maximally when there was an adjacent wall element east of the rat; and cell $5 \mathrm{p}$ fired maximally when there was a wall element $\sim 30 \mathrm{~cm}$ northwest of the rat (and perhaps also a similar distance southeast of the rat). The maximal responses of the cells were in $\operatorname{similar} x$ and $y$ offsets relative to the rat, regardless of the shape of the arena, whether it was square, round, or a square tilted on the side. When the arena was round (round is probably better for this analysis because straight walls tend to create straight strips in the plot, as noted above), a field resembling the shape of a boundary vector emerged. See for example cell $1 c$ - the field has the shape of a drop, with the pivot at the " $X$ " (the rat's position), similar to the shape of a boundary vector [compare with the shape of the boundary

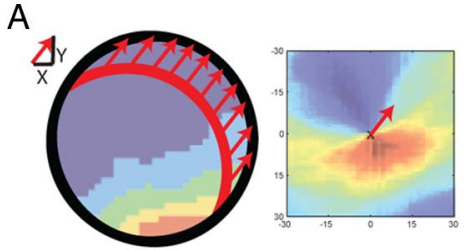

\section{B}

cell 1a
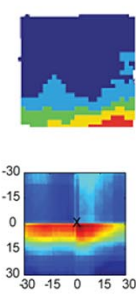

cell 1c
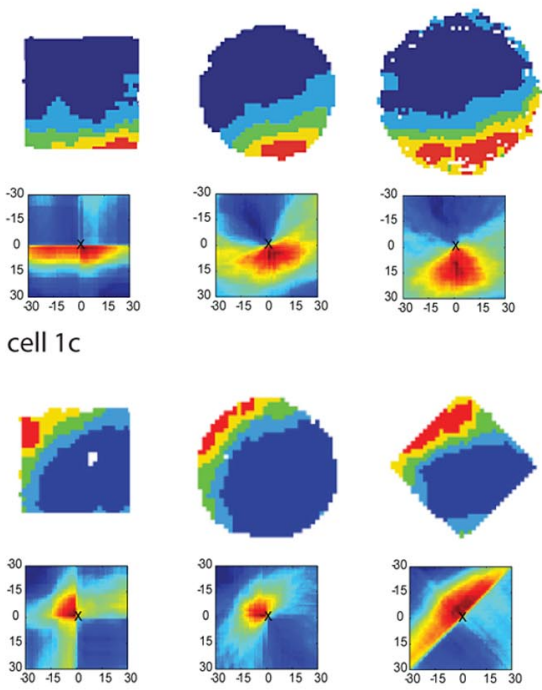

C

C

hippocampus

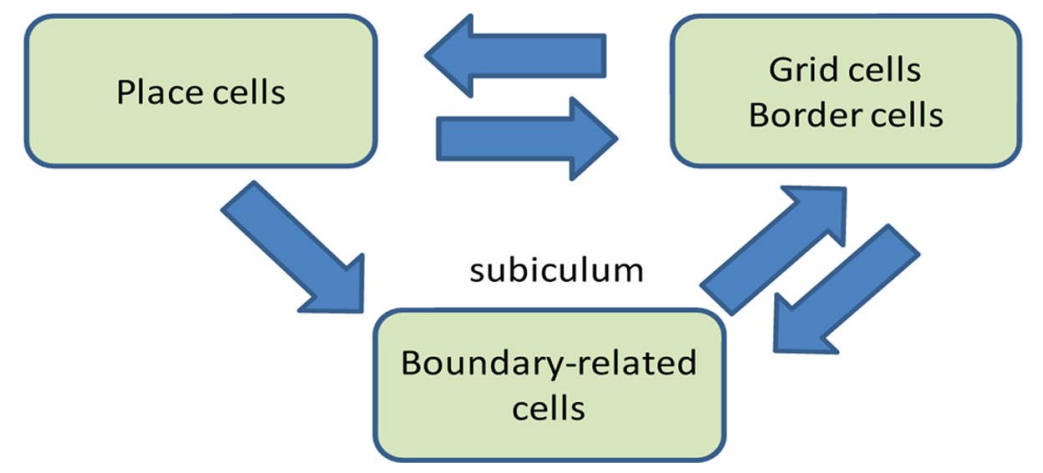

Figure 1. A, Derivation of the boundary rate plot. All pixels in the rate map with a certain $x$ and $y$ distance from a wall are marked with a red arc (left panel). The average of all these pixels contributes to a single point in the boundary rate plot (end of arrowhead; right panel), showing the mean rate of the cell when there was a wall at this $x$-distance and $y$-distance from the rat. B, Boundary rate plots resemble boundary vectors. Four examples of cells were taken from Lever et al. (2009), their Figure 3, reprinted with permission. The top row for each cell plot shows the rate maps of the cells in three different environments. The bottom row shows the corresponding calculated boundary rate plots. The " $X$ " at the center of the image is the rat's own position. Axis units are approximately in centimeters (assuming that the original images were plotted at a scale of $\sim 1 \mathrm{pixel} / \mathrm{cm}$ ). $C$, Anatomy reveals that boundary related cells in the subiculum are most likely outputs of the hippocampus, and not inputs to the hippocampus, as suggested by the original boundary-vector model. According to this sketch, boundary-related cells in the subiculum should influence border cells and grid cells directly but not place cells, while being influenced directly by place cells, grid cells and border cells.

vector in Lever et al.'s (2009) Fig. $1 B$ ]. This suggests that these cells indeed have boundary-vector properties.

As an explanation for the geometric dependency of place-cell firing (O'Keefe and Burgess, 1996), one possible caveat is that the subiculum is commonly regarded as an output structure of the hippocampal formation and not as an input structure
(Witter and Amaral, 2004; Witter, 2006). To fit their data to the boundary-vector cell model of place-cell formation, Lever et al. (2009) suggested that the subiculum can be regarded in some aspects also as an input structure of the hippocampus. However I prefer to adhere to the conventional anatomical dogma. The subiculum receives major input from CA1 without 
sending many reciprocal connections. Instead, the subiculum sends vast projections to sub-cortical and cortical structures, including the entorhinal cortex, the presubiculum and the parasubiculum. Are these suggested boundary-vector cells then actually outputs and not inputs of place cells? This is of course possible. Hartley et al. (2000) suggested constructing place cells as linear sums of boundaryvector cells, but in light of the original finding that place cell firing is determined by the rat's distance from certain walls (O'Keefe and Burgess, 1996), it may very well be the contrary: boundary-vector cells in the subiculum may be linear sums of place cell outputs, such that their properties can be derived from the properties of place-cells, and not vice-versa. Related to this, it would have been interesting if the response of subicular boundaryrelated cells in boxes in two different rooms had been checked, because it is known that place cells undergo global remapping in such circumstances (O'Keefe and Conway, 1978; Leutgeb et al., 2005). Will these boundary-responsive subicular cells continue to code for boundaries when the rat is moved from one room to another? Or would they remap their position entirely, ceasing to be boundary related?

We should not forget that there are also place-responsive cells in the entorhinal cortex. Two major classes are relevant here: grid cells (Hafting et al., 2005) and border cells (Savelli et al., 2008; Solstad et al., 2008). From an anatomical point of view, it seems reasonable that subicular boundary-related cells help to anchor grid cells to the walls, and the apparent anchoring of place cells to walls, as seen in O'Keefe and Burgess (1996), arises secondarily. Furthermore, it could very well be that the boundary-related cells in the subiculum influence the responses of border cells in the entorhinal cortex. While border cells in the MEC can fire along all walls (Solstad et al., 2008), in obvious contradiction to the concept of a boundary vector cell, they perhaps integrate information from several subicular boundaryvector cells. As Lever et al. (2009) point out, it is still not clear whether border cells have boundary-vector properties. The converse is also true, though. It is not completely clear yet whether some of the cells in the subiculum are actually border cells without boundary-vector properties. More research is needed to differentiate between these possibilities. In the meantime, I suggest that the role of the boundary-related cells in the subiculum is to pass information about boundaries, stored in the hippocampus, back to the entorhinal cortex, where the actual anchoring of the grids to the walls is likely to be performed (Fig. 1C).

In summary, the study by Lever et al. (2009) shows that at least some cells in the subiculum may have boundary vector properties. It is currently unknown who receives information from these boundaryvector cells, however it is plausible that they are used by the entorhinal cortex to help grid cells and border cells anchor to walls using the prior information the rat has about place, which is encoded in the hippocampus.

\section{References}

Hafting T, Fyhn M, Molden S, Moser MB, Moser EI (2005) Microstructure of a spatial map in the entorhinal cortex. Nature 436:801-806.

Hartley T, Burgess N, Lever C, Cacucci F, O’Keefe J (2000) Modeling place fields in terms of the cortical inputs to the hippocampus. Hippocampus 10:369-379.

Leutgeb S, Leutgeb JK, Barnes CA, Moser EI, McNaughton BL, Moser MB (2005) Independent codes for spatial and episodic memory in hippocampal neuronal ensembles. Science 309: 619-623.

Lever C, Burton S, Jeewajee A, O’Keefe J, Burgess $\mathrm{N}$ (2009) Boundary vector cells in the subiculum of the hippocampal formation. J Neurosci 29:9771-9777.

O’Keefe J, Burgess N (1996) Geometric determinants of the place fields of hippocampal neurons. Nature 381:425-428.

O’Keefe J, Conway DH (1978) Hippocampal place units in freely moving rat-why they fire where they fire. Exp Brain Res 31:573-590.

Savelli F, Yoganarasimha D, Knierim JJ (2008) Influence of boundary removal on the spatial representations of the medial entorhinal cortex. Hippocampus 18:1270-1282.

Solstad T, Boccara CN, Kropff E, Moser MB, Moser EI (2008) Representation of geometric borders in the entorhinal cortex. Science 322:1865-1868.

Witter MP (2006) Connections of the subiculum of the rat: topography in relation to columnar and laminar organization. Behav Brain Res 174:251-264.

Witter MP, Amaral DG (2004) Hippocampal formation. In: The rat nervous system, Ed 3 (Paxinos G, ed), pp 635-704. San Diego, CA: Elsevier Academic. 